\title{
Deflexión craneal y convexidad facial: Parámetros para identificar clase esqueletal previo tratamiento Ortodóntico
}

\section{Cranial deflection and facial convexity: Parameters to identify skeletal class after orthodontic treatment}

DOI: $10.46932 / \mathrm{sfjdv3n1-044}$

Received in: Dec 30st, 2021

Accepted in: Jan 1th, 2022

\section{Yolanda Azucena Guerra Mendoza}

Especialista en Radiología Dento Máxilofacial. Especialista en Ortodoncia

Universidad Internacional del Ecuador UIDE

Simón Bolívar y Jorge Fernández

E-mail: yguerra@oriongroup.ec

Miriam Fernanda Ortega López

Especialista en Docencia Clínica Odontológica, Especialista en Odontopediatría

Universidad Tecnológica Equinoccial UTE

Bartolomé Ruiz entre Gonzalo Pizarro y Conquistadores

E-mail: mfortegal38@ hotmail.com

Daniel Emanuel Ramírez Romero

Especialista en Ortodoncia

Universidad Católica de Cuenca UCACUE

Calle German león Ramírez 107

E-mail: daniel.ramrom@gmail.com

\section{Ronald Roossevelt Ramos Montiel}

Especialista en Ortodoncia, Especialista en Docencia Clínica Odontológica, PhD Doctor en Ciencias Odontológicas, PD Postdoctorado de Investigación en Ingeniería e Innovación

Universidad Católica de Cuenca UCACUE

Manuel J. Calle Y Paucarbamba

E-mail: ronald_mtz@hotmail.com

\section{RESUMEN}

Introducción: Para diagnosticar una maloclusión de clase esqueletal además de la clínica, la cefalometría de Ricketts ha sido la herramienta utilizada por excelencia. Objetivo: determinar la deflexión craneal y convexidad facial como parámetros de identificación de la clase esqueletal previo al tratamiento Ortodóntico en pacientes atendidos en la Facultad de Odontología de la Universidad de GuayaquilEcuador. Métodos: Investigación observacional, retrospectiva y transversal en muestra no probabilística de 27 pacientes en edades de 10 a 22 años a más. Se realizaron dibujos anatómicos y trazados de orientación a valores lineales y angulares. El perfil facial se determinó utilizando la medición plano estético de Ricketts. Se realizó una ficha donde se registró la deflexión craneal y la convexidad facial. Resultados: $100 \%$ de los pacientes presentaron distancia de deflexión craneal de $05 \mathrm{~mm}$ a más; en la convexidad facial el 70,3\% tenía una distancia entre 3 y $4 \mathrm{~mm} .70,3 \%$ poseía perfil recto y 59,2 tenía biotipo Dolicofacial. Conclusiones: la deflexión craneal y convexidad facial son parámetros cefalométricos que determinaron con efectiva y validez el patrón esqueletal de los individuos de este 
estudio para establecer el tratamiento ortodóntico que responda a las expectativas del paciente y del clínico.

Palabras clave: Deflexión craneal, convexidad facial, clase esqueletal.

\begin{abstract}
Introduction: Ricketts cephalometry has been the tool used par excellence to diagnose a skeletal class malocclusion in addition to the clinical one. Objective: to determine the cranial deflection and facial convexity as identification parameters of the skeletal class prior to Orthodontic treatment in patients treated at the Faculty of Dentistry of the University of Guayaquil-Ecuador. Methods: Observational, retrospective and cross-sectional research in a non-probabilistic sample of 27 patients aged 10 to 22 years and older. Anatomical drawings and orientation traces were made at linear and angular values. The facial profile was determined using the Ricketts aesthetic plane measurement. A card was made where the cranial deflection and facial convexity were recorded. Results: $100 \%$ of the patients presented a cranial deflection distance of $05 \mathrm{~mm}$ or more; in facial convexity, $70.3 \%$ had a distance between 3 and $4 \mathrm{~mm}$. $70.3 \%$ had a straight profile and 59.2 had a Dolichofacial biotype. Conclusions: cranial deflection and facial convexity are cephalometric parameters that effectively and validly determined the skeletal pattern of the individuals in this study to establish orthodontic treatment that responds to the expectations of the patient and the clinician.
\end{abstract}

Keywords: Cranial deflection, facial convexity, skeletal class.

\title{
1 INTRODUCCIÓN
}

Las maloclusiones son alteraciones o desórdenes oclusales sujetos a importantes condicionantes estéticos, étnicos y culturales, estas van desde la función masticatoria y trastornos de la articulación temporomandibular hasta alteraciones de la columna vertebral ${ }^{1}$.

La Organización Mundial de la Salud (OMS) señala que las maloclusiones constituyen un problema de Salud Pública, ocupando el tercer lugar entre los problemas de salud bucodental a nivel mundial $^{2}$. Su prevalencia es muy variable y amplia estimándose entre $39 \%$ y $93 \%$ en niños y adolescentes, la ausencia de heterogeneidad pudiese ser atribuidas a diferencias étnicas y de edad. ${ }^{3}$

Se identifican cuando los controles anatomo-fisiológicos del sistema estomatognático se encuentran en desarmonía con los segmentos dentarios, pudiendo estar presentes desde el origen del individuo, o bien durante las primeras etapas de vida, siendo de magnitudes diferentes con el paso del tiempo ${ }^{4}$. Son ocasionadas por múltiples factores, como la herencia, las diferencias maxilo-mandibulares del crecimiento y las malposiciones dentarias en cada arcada y en estas influyen, las alteraciones en la cronología de la erupción dentaria, la pérdida prematura de dientes y la caries dental entre otros ${ }^{1}$

Las maloclusiones pueden ocurrir en tres planos espaciales diferentes sagital, transversal y vertical. Es posible identificar tres tipos diferentes de relación esquelética en el plano sagital, definidos a partir del análisis del ángulo ANB, que representa la relación intermaxilar anteroposterior ${ }^{3}$. Edward Angle $^{5}$ fue quien realizó la primera clasificación ortodóntica de maloclusión siendo estas clases I, II y III; 
se basó en la hipótesis de que el primer molar y canino son los dientes más estables de la dentición y la referencia de la oclusión.

De acuerdo a esta clasificación la oclusión clase I se caracteriza por las relaciones mesiodistales normales de los maxilares y arcos dentales, indicada por la oclusión normal de los primeros molares. En promedio los arcos dentales están ligeramente colapsados, con el correspondiente apiñamiento de la zona anterior la maloclusión está confinada principalmente a variaciones de la línea de oclusión en la zona de incisivos y caninos 5 .

La oclusión clase II ocurre cuando por cualquier causa los primeros molares inferiores ocluyen distalmente a su relación normal con los primeros molares superiores en extensión de más de una mitad del ancho de una cúspide de cada lado y sucesivamente los demás dientes ocluirán anormalmente y estarán forzados a una posición de oclusión distal, causando más o menos retrusión o falta de desarrollo de la mandíbula 5 .

La maloclusión clase III es aquella caracterizada por la oclusión mesial de ambas hemiarcadas del arco dental inferior hasta la extensión de ligeramente más de una mitad del ancho de una cúspide de cada lado. Puede existir apiñamiento de moderado a severo en ambas arcadas, especialmente en el arco superior. Existe inclinación lingual de los incisivos inferiores y caninos, la cual se hace más pronunciada entre más severo es el caso, debido a la presión del labio inferior en su intento por cerrar la boca y disimular la maloclusión. El sistema neuromuscular es anormal encontrando una protrusión ósea mandibular, retrusión maxilar o ambas. ${ }^{5}$

Proffit $^{6}$ describe la maloclusión tipo III, como una relación en la cual los incisivos y/o caninos del maxilar superior se encuentran en posición lingual con respecto a sus homólogos mandibulares, mientras que Graber $^{7}$ la describe como verdaderas displasias dentoesqueletales, donde se reconoce una relación anteroposterior de los maxilares en relación con la base del cráneo, estando presentes o no irregularidades de los dientes.

La etiología es multifactorial y se asocia con factores hereditarios e influencias ambientales, dando lugar a maloclusiones de tipo dental, esqueletal o funcional, haciendo su diagnóstico y abordaje muy complejo $^{8}$

Para diagnosticar una maloclusión de clase esqueletal además de la clínica, la cefalometría de Ricketts $^{9}$ ha sido la herramienta por excelencia utilizada, por medio de ella se obtienen los valores como convexidad facial, profundidad maxilar, profundidad facial, deflexión craneal, base craneal anterior, base craneal posterior, localización del porio, longitud cuerpo mandibular, ángulo ANB, ángulo goníaco, línea estética E. ${ }^{10}$ 
Respecto a la convexidad facial, la cefalometría de Ricketts ${ }^{9}$ la define como la distancia medida en milímetros desde el punto A al plano facial (Na-Po). Esta distancia presenta una norma clínica donde a los 9 años el valor debe ser de $+2 \mathrm{~mm}$ disminuyendo $0,2 \mathrm{~mm}$ por año con un DS $\pm 2 \mathrm{~mm}$. Atendiendo a estas medidas y sus variaciones con la edad se establece la clase esqueletal. Si este valor del punto A al plano facial es inferior a los $2 \mathrm{~mm}$ se considerará al paciente como portador de Clase III esqueletal ${ }^{10}$.

Por otra parte la deflexión facial es definida como el ángulo que se mide entre Basión- Nasión y el plano de Frank-fort. Es una valoración de estructuras internas donde hereditaria y genéticamente nos orienta sobre patrones de crecimiento dominantes. Su norma es de $27^{\circ} \pm 3^{\circ}$. Un ángulo aumentado, mayor a $20^{\circ}$, nos indica que la cavidad glenoidea se encuentra adelantada lo que complica la situación de patrón de crecimiento de clase III $^{10}$.

Mora et $\mathrm{al}^{8}$ sostienen que el análisis cefalométrico resulta esencial para confirmar el diagnóstico presuntivo, determinar el origen real de la anomalía y permitir formular un plan de tratamiento adecuado, quirúrgico o no-quirúrgico, que responda a las exigencias del paciente y el clínico.

El objetivo de esta investigación fue determinar la deflexión craneal y convexidad facial como parámetros de identificación de la clase esqueletal previo al tratamiento Ortodóntico en pacientes atendidos en la Facultad Piloto de Odontología Dr. José Apolo Pineda de la Universidad de GuayaquilEcuador.

\section{MATERIALES Y MÉTODOS}

Investigación descriptiva, observacional, retrospectiva y transversal. Se utilizaron radiografías cefalométricas laterales de cráneo, de 27 pacientes atendidos durante el periodo 2013-2015 en la Facultad de Odontología Dr. José Apolo Pineda de la Universidad de Guayaquil- Ecuador.

Se realizaron dibujos anatómicos y los trazados de orientación, a los valores lineales y angulares. Las medidas angulares se realizaron con la ayuda de un transferidor, expresándolas en grados de ángulo. Las medidas lineales se utilizaron como unidad en milímetro y se calcularon mediante una regla milimetrada.

Para facilitar la lectura e interpretación de los datos, se estandarizó la representación de los ángulos para escanearlos. Para analizar y determinar qué tipo de perfil facial se utilizó la medición plano estético de Ricketts $^{9}$ y se realizó una ficha de recolección de datos donde se registró la deflexión craneal, y la convexidad facial para establecer la clase esqueletal.

\subsection{ANÁLISIS ESTADÍSTICO}

El procesamiento estadístico se realizó utilizando el programa SPSS versión 19.0 de IBM $^{\circledR}$. Se utilizó estadística descriptiva para el análisis de los datos. 


\section{ASPECTOS ÉTICOS}

La investigación fue aprobada por el comité de Bioética de la Universidad de Guayaquil- Ecuador para el consentimiento del uso de los datos registrados en las historias clínicas de los pacientes atendidos durante el periodo 2013-2015 dando garantía de la confidencialidad de la información proporcionada.

\section{RESULTADOS}

El 48\% (13) de los individuos de la muestra se ubicó en el grupo de 22 años de edad a más de edad, seguido del grupo de 10 a 15 años con el 29,7 (8) y el grupo de 16 a 21 años con el 22,2\% (6). El 55,5\% (15) pertenecía al género masculino y el 44,4\% (12) al femenino.

El 100\% (27) de los pacientes presentó una distancia de deflexión craneal de 05 mm a mas, mientras que en la convexidad facial el mayor porcentaje se presentó en el 70,3\% de los individuos con una distancia entre 3 y $4 \mathrm{~mm}$. (Tabla 1).

En cuanto a la distribución de la muestra según el perfil y biotipo facial, se determinó que 70,3\% (19) poseía perfil recto y el 59,2(16) tenía biotipo Dolicofacial (tabla 2).

Los cambios en los valores la deflexión craneal y convexidad facial y su identificación según la clase esqueletal se muestran en la tabla 3.

Tabla 1 Distancia de deflexión craneal y convexidad facial de los pacientes de la muestra en estudio

\begin{tabular}{|c|c|c|c|}
\hline Distancia (Ricketts) & $\mathrm{n}=27$ & $\%$ & $=100$ \\
\hline \multicolumn{4}{|l|}{ Deflexión Craneal } \\
\hline 01 a $02 \mathrm{~mm}$ & 00 & & 00 \\
\hline 03 a $04 \mathrm{~mm}$ & 00 & & 00 \\
\hline 05 a más mm & 27 & & 100 \\
\hline \multicolumn{4}{|l|}{ Convexidad facial } \\
\hline 01 a $02 \mathrm{~mm}$ & 08 & & 29,6 \\
\hline $\begin{array}{l}03 \text { a } 04 \mathrm{~mm} \\
05 \text { a más mm }\end{array}$ & $\begin{array}{l}19 \\
00\end{array}$ & & $\begin{array}{l}70,3 \\
00,0\end{array}$ \\
\hline
\end{tabular}

Fuente: Historia Clínica

Tabla 2. Perfil y biotipo facial de los pacientes de la muestra en estudio $\mathrm{n}=27 \quad \%=100$

$\begin{array}{lll}\text { Perfil Facial } & & \\ \text { Convexo } & 05 & 18,5 \\ \text { Recto } & 19 & 70,3 \\ \text { Concavo } & 03 & 11,1 \\ & & \\ \text { Biotipo facial } & & \\ \text { Braquifacial } & 05 & 18,3 \\ \text { Mesofacial } & 06 & 22,2 \\ \text { Dolicofacial } & 16 & 59,2\end{array}$

Fuente: Historia Clínica 
Tabla 3. Deflexión craneal, convexidad facial e identificación de la clase esqueletal de la muestra en estudio

\begin{tabular}{clll}
\hline Valores & $\mathrm{n}$ & $\%$ & Clase Esqueletal \\
\hline Deflexión craneal & 05 & 18,3 & \\
Aumentado & 19 & 70,3 & Clase III \\
Se mantiene & 03 & 11,1 & Clase I \\
Disminuido & & & Clase II \\
Convexidad Facial & 14 & 51,8 & Clase II \\
Aumentada & 10 & 37,3 & Clase I \\
Se mantiene & 03 & 11,1 & Clase III \\
Disminuido & &
\end{tabular}

Fuente: Historia Clínica

\section{DISCUSIÓN}

Este estudio determinó la deflexión craneal y convexidad facial como parámetros para establecer de la clase esqueletal previo al tratamiento Ortodóntico en pacientes atendidos en la Facultad Piloto de Odontología Dr. José Apolo Pineda de la Universidad de Guayaquil- Ecuador.

En cuanto a la deflexión craneal todos los individuos de este estudio reportaron valores de deflexión craneal de $05 \mathrm{~mm}$ a Almeida et al ${ }^{11}$ plantearon que el ángulo de la base del cráneo no parece desempeñar un rol clave en el desarrollo de las clases esqueletales, siendo relativamente estable en edades de 5 a 15 años. El mayor número de pacientes de esta investigación pertenecían al grupo de edad de 22 años a mas por lo que ya han culminado su proceso de crecimiento y desarrollo.

Ruiz et al ${ }^{12}$ postulan que una variación que hay que tomar en cuenta es la longitud de la base craneal posterior la cual puede compensar cualquier deflexión craneal. Por ejemplo, el efecto de un ángulo de la base craneal cerrado que ubicará a la cavidad glenoidea y mandíbula en una posición más anterior, podrá ser contrarrestado por una longitud de la base craneal posterior aumentada, la cual desplazará al punto articular y por consiguiente a la mandíbula a una posición más posterior.

Respecto a la convexidad facial el mayor porcentaje de individuos presentaron valores ubicados entre 3 y $4 \mathrm{~mm}$. Si el valor del punto A al Plano Facial es inferior a los $2 \mathrm{~mm}$ se considerará al paciente como portador de Clase III esqueletal. Pérez et $\mathrm{al}^{13}$ señalan que la convexidad de Ricketts considera la posición sagital de un maxilar en relación al otro y no en base a un referente craneal, lo cual, se puede traducir en que pacientes clase I presenten ambos maxilares adelantados en igual medida, de manera que el perfil se observa biprotruido, también debe considerarse la variación propia de cada cultura o etnia que modifican las preferencias estéticas.

Los pacientes estudiado presentaron predominantemente un perfil facial recto y en lo referente al biotipo facial hubo mayor prevalencia del biotipo Dolicofacial, este perfil posee mandíbulas con ramas poco desarrolladas en relación al cuerpo, cara alargada, arcadas dentarias angostas y Presenta divergencia 
entre la mandíbula y la base craneal y entre la base mandibular y el plano biespinal predominando el largo sobre el ancho en las dimensiones esqueletalesal ${ }^{14}$ Estos resultados son similares a los reportados por Inda-Velasquez et $\mathrm{al}^{15}$ quienes encontraron que este perfil fue el predominante en individuos de su estudio y difiere de lo reportado por Querales et $\mathrm{al}^{16}$ quienes encontraron mayor prevalencia de perfil facial convexo en una región de Venezuela. El biotipo del paciente afecta directamente la armonía facial, los músculos orofaciales, la oclusión y la función estomatognática, lo cual hace que sea importante determinarlo para cualquier tratamiento ortodóntico ${ }^{17}$.

La biotipología se encuentra directamente relacionada con el crecimiento y el cambio de la forma de la base ósea orofacial, es decir los huesos maxilares, dientes y articulación temporomandibulares. ${ }^{18}$ por lo que el desconocimiento del biotipo facial y esqueletal puede ocasionar errores en el diagnóstico y por ende en el plan de tratamiento ya que los biotipos faciales presentan distintas respuestas frente a fuerzas ortodónticas similares ${ }^{19}$

Con respecto a la distribución de sujetos según la clase esqueletal basado en la deflexión craneal, se encontró que los valores de la misma se mantuvieron en los rangos establecidos en la mayoría de los individuos de este estudio lo que los ubicaría en el patrón esqueletal clase I; sin embargo en estos mismos individuos se encontró que solo un porcentaje menor se mantuvo en la norma en cuanto a la convexidad facial.

Así mismo un porcentaje menor de sujetos se ubicó en la clase esqueletal III, según el parámetro deflexión craneal el cual se reportó aumentado, aun cuando un porcentaje menor tenia los valores de la convexidad facial disminuida. Se ha postulado que la maloclusión clase III esqueletal cefalométricamente está caracterizada por convexidad facial disminuida a expensas de un valor inferior de la profundidad maxilar, al aumento de la profundidad maxilar o a ambas situaciones a la vez. Una posible explicación pudiera tener su base en que según Ricketts ${ }^{9}$ la distancia medida en milímetros desde el punto A al plano facial presenta una norma clínica donde a los 9 años el valor debe ser de $+2 \mathrm{~mm}$ disminuyendo 0,2 $\mathrm{mm}$ por año con un DS $\pm 2 \mathrm{~mm}$ y la muestra de este estudio fue mayoritariamente de 22 años a más. Atendiendo a estas medidas y sus variaciones con la edad se establece la clase esqueletal. Si este valor del punto A al plano facial es inferior a los $2 \mathrm{~mm}$ se considerará al paciente como portador de Clase III esqueletal ${ }^{10}$.

Se concluye que la deflexión craneal y convexidad facial son parámetros cefalometricos que determinaron con efectiva y validez el patrón esqueletal de los individuos de este estudio para diseñar y establecer un plan de tratamiento ortodontico que responda a las expectativas del paciente y del clínico. 


\section{REFERENCIAS}

1. Aguilar Moreno, Norma Angélica, Taboada Aranza, Olga. (2013). Frecuencia de maloclusiones y su asociación con problemas de postura corporal en una población escolar del Estado de México. Boletín médico del Hospital Infantil de México, 70(5), 64-371.

2. Organización Mundial de la Salud. Salud bucodental. Nota informativa. (2012) ํ318.

3. Cenzato N, Nobili A, Maspero C. Prevalence of Dental Malocclusions in Different Geographical Areas: Scoping Review. Dent J (Basel). 2021 Oct 11;9(10):117. doi: 10.3390/dj9100117

4. Espinal-Botero G, Muñoz A, Flores L, Ponce M, Nava J, González J.(2016) Frecuencia de maloclusión en las clínicas odontopediátricas de la Universidad de Antioquia, Colombia, y de la Universidad Autónoma de San Luis Potosí, México. Rev Nac Odontol. 2016:12(22):61-68

5. Angle EH.Classification of the malocclusion. Dental Cosmos. 1889.41: 2 48-264

6. Proffit W. Ortodoncia teoría y práctica. 2007. Editorial Mosby. $4^{\text {a }}$ edición

7. Graber TM. (1981).Ortodoncia teoría y práctica: Ed. Interamericana, tercera edición.; Capítulo 6 y 7: 239-374.

8. Mary Alejandra Mora Martínez, Roberto Pesqueira Melgarejo, Guillermo Hernández Espinosa, José Luis De Silva Dávila, Jacqueline Adelina Rodríguez Chávez. Class III dentofacial alteration treated with camouflage: Clinical case report. Revista Mexicana de Ortodoncia.2015; 3(1): 62-69

9. Ricketts, R (1961). Cephalometric Analysis And Synthesis. The Angle Orthodontist.1962. 31(3): 141-156.

10. Lorena Y Palczikowski, Carmen I Collante de Benitez . Diagnóstico de Clase III: Identificación del patrón esqueletal. Revista Facultad de Odontologia. 2016.9(1):50-54

11. Almeida KCM, Raveli TB, Vieira CIV, et al. Influence of the cranial base flexion on Class I, II and III malocclusions: a systematic review. Dent Press J Orthod. 2017;22(5):56-66. Doi: 10.1590/21776709.22.5.056-066.oar.

12. Ruiz Rivera Aldo, Canseco Jiménez Joaquín, Cuairán Ruidíaz Vicente. Relación entre la deflexión de la base del cráneo y la clase ósea Rev. Odont. Mex.2011.15 (4)

13. Pérez-G Marcelo, Carreño-L Susana, Araya-Díaz Pamela, Parada-I Juan Guillermo, Palomino-M Hernán, Martínez-R Vicente et al . Determinación de Valores de Armonía del Perfil Facial en la Población Chilena. Int. J. Odontostomat. 2021 ; 15( 1 ): 137-144.. $\quad$ Doi: http://dx.doi.org/10.4067/S0718$381 X 2021000100137$.

14. Cerda-Peralta B, Schulz-Rosales R, López-Garrido J, Romo- Ormazabal F. Parámetros cefalométricos para determinar biotipo facial en adultos chilenos. Rev Clin Periodoncia Implantol Rehabil Oral. 2019; 12(1): 8-11.

15. Inda-Velázquez KL, Gutiérrez-Rojo JF, Gutiérrez-Villaseñor J. Relación del biotipo facial determinado con el VERT y el patrón de crecimiento facial . Oral. 2019;20(64):1762-1765 
16. Querales Alvarado Marianna. ,García Alvarado Mariangel, Maldonado Villamizar Johana María. Compromiso de la línea estética de Ricketts y el perfil facial en pacientes con mordida abierta sin crecimiento. Revista latinoamericana de ortodoncia y odontopediatria.2015 https://www.ortodoncia.ws/publicaciones/2015/art-40/

17. Arciniega, M. Y. Estudio Piloto: Medidas mndibulares de los diferentes biotipos faciales en poblacion infantil mexicana de 6 años de edad, residente en la ciudad de Mexico. Rev. Odontologica Mexicana 2009: 13 (3): 141-147.

18. Zamora, C. I. Atlas de Cefalometria . AMOLCA. 2a Edición. Colombia.2010.

19. Menéndez, Méndez, L. V. Estudios cefalométricos en diferentes poblaciones mediante el análisis de Ricketts. Rev Odontología Sanmarquina.2008.11 (1): 22-24. 